\title{
QUEDA DO MESMO NÍVEL EM IDOSOS: FATORES ASSOCIADOS AO TRAUMA CRANIOENCEFÁLICO E RAQUIMEDULAR*
}

\author{
Josy Anne Silva ${ }^{1}$, Gustavo Henrique Marques Moreno ${ }^{2}$, Liliana Yukie Hayakawa ${ }^{3}$, Kelly Cristina Inoue \\ Roberto Kenji Nakamura Cuman ${ }^{4}$
}

\begin{abstract}
RESUMO: Objetivo: identificar fatores associados à ocorrência de trauma cranioencefálico e raquimedular entre idosos que sofreram queda do mesmo nível. Método: estudo retrospectivo, com 192 prontuários de idosos que sofreram queda do mesmo nível em 2014 e foram atendidos no Pronto Atendimento de um hospital de ensino do Paraná. Realizou-se teste $Z$, para comparação de proporções do grupo com $(n=80)$ e sem $(n=112)$ trauma cranioencefálico ou raquimedular com dados demográficos, de saúde, da queda e da avaliação/atendimento. Resultados: dos idosos identificados, 80 (41,7\%) tiveram traumatismo cranioencefálico ou raquimedular. Houve associação significativa com a ocorrência de traumatismo cranioencefálico ou raquimedular: sexo masculino $(p=0,0109)$, transporte especializado ( $p=0,0001)$, queda em via pública ( $p=0,0026)$, ausência de hipertensão arterial $(p=0,0434)$ e presença de doença psiquiátrica $(p=0,0048)$. Conclusão: o trauma cranioencefálico ou raquimedular é um evento frequente associado à queda do mesmo nível, o que denota a necessidade de medidas educativas e preventivas visando à redução da quedas.
\end{abstract}

DESCRITORES: Serviços de saúde para idosos; Envelhecimento; Traumatologia; Ferimentos e lesões; Medicina de emergência.

\section{SAME-LEVEL FALLS IN OLDER ADULTS: FACTORS ASSOCIATED WITH TRAUMATIC BRAIN AND SPINAL CORD INJURIES}

\begin{abstract}
Objective: to identify factors associated with the occurrence of traumatic brain and spinal cord injuries among older adults who suffered a same-level fall. Method: a retrospective study, with 192 medical records of older adults that suffered a samelevel fall in 2014 and were attended in the Emergency Care Unit of a teaching hospital in Paraná. The Z test was performed to compare the proportions of the groups with $(n=80)$ and without $(n=112)$ traumatic brain or spinal cord injuries with demographic, health, fall and evaluation/care data. Results: $80(41.7 \%)$ of the older adults identified had traumatic brain or spinal cord injuries. There was a significant association of the occurrence of traumatic brain or spinal cord injuries with: male gender ( $\mathrm{p}=0.0109)$, specialized transport $(p=0.0001)$, fall on public road $(p=0.0026)$, absence of arterial hypertension $p=0.0434)$ and presence of psychiatric illness $(p=0.0048)$. Conclusion: traumatic brain and spinal cord injuries are frequent events associated with same-level falls, which indicates the need for educational and preventive measures aimed at the reduction of falls.
\end{abstract}

DESCRIPTORS: Health services for the older adults; Aging; Traumatology; Wounds and injuries; Emergency medicine.

\section{CAÍDA DESDE ALTURA SIMILAR EN ANCIANOS: FACTORES ASOCIADOS AL TRAUMA CRANEOENCEFÁLICO Y RAQUIMEDULAR}

RESUMEN: Objetivo: Identificar factores asociados a la ocurrencia de traumas craneoencefálicos y raquimedulares entre ancianos que sufrieron caídas desde alturas similares. Método: Estudio retrospectivo, con 192 historias clínicas de ancianos que cayeron desde alturas similares en 2014, atendidos en la Guardia de un hospital de enseñanza de Paraná. Se realizó test Z para comparación de proporciones del grupo con $(n=80)$ y $\sin (n=112)$ trauma craneoencefálico o raquimedular con datos demográficos, de salud, de la caída y de la evaluación/atención. Resultados: Ochenta $(41,7 \%)$ ancianos sufrieron traumatismo craneoencefálico o raquimedular. Existió asociación significativa con traumatismo craneoencefálico o raquimedular en: sexo masculino ( $p=0,0109$ ), transporte especializado $(p=0,0001)$, caída en vía pública $(p=0,0026)$, ausencia de hipertensión arterial $(p=0,0434)$ y presencia de enfermedad psiquiátrica $(p=0,0048)$. Conclusión: El trauma craneoencefálico o raquimedular constituye un evento frecuentemente asociado a caídas desde niveles similares, expresando ello necesidad de medidas educativas y preventivas apuntando a reducir las caídas.

DESCRIPTORES: Servicios de Salud para Ancianos; Envejecimiento; Traumatología; Heridas y Lesiones; Medicina de Emergencia.

\footnotetext{
*Artigo extraído da dissertação de mestrado "Associação entre o uso de medicamentos e os fatores de risco para queda em idosos portadores de doenças crônico degenerativas atendidos no Pronto Atendimento de um Hospital Universitário público referência no noroeste do Paraná". Universidade Estadual de Maringá, 2016.
}

${ }^{1}$ Enfermeira. Mestre em Ciências da Saúde. Universidade Estadual de Maringá. Maringá, PR, Brasil.

${ }^{2}$ Fisioterapeuta. Mestrando em Ciências da Saúde. Universidade Estadual de Maringá. Maringá, PR, Brasil.

${ }^{3}$ Enfermeira. Doutora em Enfermagem. Universidade Estadual de Maringá. Maringá, PR, Brasil.

${ }^{4}$ Farmacêutico. Doutor em Farmacologia. Docente da Universidade Estadual de Maringá. Maringá, PR, Brasil.

Autor Correspondente:

Recebido: $14 / 11 / 2017$

Roberto Kenji Nakamura Cuman

Finalizado: $22 / 08 / 2018$

Universidade Estadual de Maringá

Av. Colombo, 5790 - 87020-900 - Maringá, PR, Brasil

E-mail: rkncuman1@gmail.com 


\section{INTRODUÇÃO}

O aumento da população idosa é um fato relevante e que requer atenção. No Brasil, a população de idosos (60 anos ou mais) representa 10,8\%, correspondendo a mais de vinte milhões e meio de pessoas $^{(1)}$. O avançar da idade, acrescido da longevidade, traz mudanças físicas e mentais que levam ao aumento do risco de queda(2). Contudo, a queda é um evento multifatorial e, nesse sentido, estudos de alguns autores ${ }^{(3-5)}$ têm apontado vários fatores de risco, bem como as consequências da ocorrência de quedas.

A ocorrência de queda está relacionada a uma incompatibilidade entre a função fisiológica do indivíduo, o ambiente e fatores comportamentais ${ }^{(2)}$, ou seja, são vários os fatores de risco para queda de idosos: idade avançada, sexo feminino, fragilidade física, fraqueza muscular, marcha instável e equilíbrio, cognição prejudicada, deficiência nutricional, sono irregular, deficiência visual, presença de doenças crônicas com consequente uso de medicamentos, dentre outros ${ }^{(4,6)}$.

Estima-se que, anualmente, um terço dos idosos sofrem algum tipo de queda, e destes cerca de $10 \%$ a $15 \%$ não conseguem suportar a lesão ${ }^{(7)}$ evoluindo para o óbito. Dentre as lesões mais preocupantes estão as fraturas de fêmur, o trauma cranioencefálico (TCE) e trauma raquimedular (TRM), devido à gravidade das consequências e ao impacto à saúde da vítima, com maior possibilidade de incapacidade temporária ou permanente.

Os custos da assistência para idosos que sofrem queda e ficam com sequelas são grandes. Além da imobilidade na realização das atividades cotidianas, a ocorrência de quedas desencadeia problemas financeiros para o sistema de saúde pública e para os familiares responsáveis pelo tratamento do idoso ${ }^{(8)}$. Desse modo, a identificação dos fatores de risco para a queda, bem como o monitoramento do tipo, gravidade, incidência e prevalência de quedas, são importantes para a elaboração e implementação de medidas preventivas e protocolos de atendimento que tornem a assistência mais eficiente ${ }^{(9)}$.

Alguns estudos nacionais analisaram a ocorrência do TCE e TRM, no perfil de morbimortalidade da população brasileira idosa, e identificaram a queda ${ }^{(10-11)}$ como uma de suas principais causas.

Ante ao exposto, este estudo teve como objetivo identificar os fatores associados à ocorrência de TCE ou TRM entre idosos que sofreram queda da própria altura.

\section{MÉTODO}

Estudo transversal, retrospectivo, de natureza quantitativa, desenvolvido na Unidade de Pronto Atendimento (PA) de um hospital público de ensino do Paraná. Os estudos transversais possibilitam visualizar a situação de uma população em um determinado momento ${ }^{(12)}$. O PA tem funcionamento ininterrupto e adota o sistema de Acolhimento com Classificação de Risco (ACCR), uma estratégia para organizar o atendimento dos serviços de emergência, com priorização de casos mais graves ${ }^{(13)}$ para o ingresso dos pacientes nos serviços de saúde.

Amostra não probabilística, por conveniência, selecionada a partir de relatório emitido pelo Sistema de Gestão da Assistência de Saúde (GSUS-PR), com todos os pacientes que sofreram queda no ano de 2014. Como critérios de inclusão foram considerados pacientes idosos (com 60 anos ou mais), que sofreram queda do mesmo nível (tropeções ou escorregões), atendidos no PA e com abertura de Autorização de Internação Hospitalar $(\mathrm{AIH})$, já que a ficha de atendimento inicial nem sempre era vinculada à abertura de prontuário do paciente para a obtenção dos dados. Como critério de exclusão, considerou-se a ausência de AIH.

Foram identificados 859 atendimentos decorrentes de queda no PA no período investigado. Destes, 627 foram excluídos por não serem idosos, 21 por serem idosos que sofreram queda de nível, cinco idosos que não tiveram nenhum tipo de lesão e 14 idosos cujos dados se encontravam incompletos (caracterização, nível da queda ou complicação da queda), totalizando 192 participantes que tiveram queda do mesmo nível, dos quais 80 tiveram TCE e/ou TRM, cujos prontuários foram localizados e utilizados na coleta de dados. 
Pacientes que atenderam aos critérios de inclusão foram dicotomizados em com ou sem TCE e/ou TRM para viabilizar testes de associação estatística, e assim, alcançar o objetivo proposto.

Utilizou-se um instrumento estruturado elaborado para esta pesquisa, contendo dados de caracterização demográfica (sexo e idade), da história de saúde (comorbidades, uso de medicamento domiciliar e queda pregressa), da queda (local, causa, período de ocorrência e primeiro atendimento), da avaliação inicial no PA (classificação de risco), e do atendimento (tipo de lesão, tempo de internação e desfecho do caso).

Os dados foram digitados em planilha do Microsoft Excel 2010 e analisados estatisticamente com o auxílio do programa Statistica Single User versão 13.2. Para as variáveis qualitativas, foram utilizadas tabelas de frequências absoluta e percentual seguidas do teste $Z$ para comparação de proporções. Para a descrição do tempo de internação, foi utilizada média com comparação do tempo de internação entre os grupos com e sem TCE/TRM. O nível de significância adotado nos testes estatísticos foi de 5\%, ou seja, foram consideradas significativas as comparações cujo p-valor era menor ou igual a 0,05.

Todos os preceitos éticos e legais vigentes foram respeitados e o projeto desta pesquisa foi aprovado pelo Comitê de Ética em Pesquisa, parecer $n^{\circ}$ 1.055.333.

\section{- RESULTADOS}

Participaram 192 idosos, 121 (63\%) do sexo feminino e 71(36,9\%) masculino, cuja idade variou de 60 anos a 90 ou mais; com tempo de internação de 1 a 57 dias (sem TCE/TRM: média $=5 \pm 7,7$ dias e com TCE/ TRM: média $=3,9 \pm 3,8$ dias).

Pacientes com TCE/TRM chegaram ao PA em transporte especializado 56 (70\%) e foram classificados em sua maioria, em amarelo 53(66,3\%).

Na Tabela 1, verifica-se a associação de variáveis de atendimento inicial com a ocorrência (ou não) de TCE/TRM.

Tabela 1 - Caracterização do atendimento inicial aos idosos que sofreram queda do mesmo nível e foram atendidos no PA. Maringá, PR, Brasil, 2014

\begin{tabular}{lccc} 
Variáveis & $\begin{array}{c}\text { Sem TCE/TRM } \\
\mathbf{n}(\%)\end{array}$ & $\begin{array}{c}\text { Com TCE/TRM } \\
\mathbf{n}(\%)\end{array}$ & p-valor \\
\hline Transporte ao PA & & & \\
\hline Não especializado $^{\mathrm{a}}(\mathrm{n}=103)$ & $79(70,5)$ & $24(30)$ & $0,0001^{*}$ \\
\hline Especializado $^{\mathrm{b}}(\mathrm{n}=89)$ & $33(29,5)$ & $56(70)$ & $0,0001^{*}$ \\
\hline Classificação de risco $^{\mathrm{c}}$ & & & \\
\hline Urgência maior - Amarelo $(\mathrm{n}=114)$ & $61(54,5)$ & $53(66,3)$ & 0,101 \\
\hline Urgência menor - Verde $(\mathrm{n}=48)$ & $38(33,9)$ & $10(12,5)$ & $0,0007^{*}$ \\
\hline Emergência - Vermelho $(\mathrm{n}=2)$ & $1(0,9)$ & $1(1,3)$ & 0,790 \\
\hline Não consta $(\mathrm{n}=28)$ & $12(10,7)$ & $16(20)$ & 0,072
\end{tabular}

* p-valor significativo pelo teste Z considerando nível de significância de 5\%.

anclui veículo próprio, acompanhamento por familiares ou transporte coletivo.

bInclui viatura do SIATE ou SAMU.

'Classificação registrada no prontuário do paciente

Na Tabela 2, no teste de associação para ocorrência de TCE/TRM em idosos com queda do mesmo nível entre pacientes com TCE/TRM, predominou a faixa etária de 60 a 70 anos 36 (45\%), queda no domicílio 55(68,8\%) e alta na maioria dos casos 77 (93,6\%); nenhuma doença crônica teve frequência elevada. 
Tabela 2 - Distribuição dos fatores associados ao TCE e TRM em idosos que sofreram queda do mesmo nível atendidos no PA. Maringá, PR, Brasil, 2014

\begin{tabular}{|c|c|c|c|}
\hline Fatores $^{\mathrm{a}}$ & $\begin{array}{c}\text { Sem TCE/TRM } \\
n(\%) \\
\end{array}$ & $\begin{array}{c}\text { Com TCE/TRM } \\
n(\%) \\
\end{array}$ & p-valor \\
\hline \multicolumn{4}{|l|}{ Faixa etária } \\
\hline De 60 a 70 anos $(n=81)$ & $45(40,2)$ & $36(45)$ & 0,507 \\
\hline De 71 a 80 anos $(n=67)$ & $43(38,4)$ & $24(30)$ & 0,229 \\
\hline De 81 a 90 anos $(n=37)$ & $19(17)$ & $18(22,5)$ & 0,341 \\
\hline 91 ou mais $(n=7)$ & $5(4,5)$ & $2(2,5)$ & 0,467 \\
\hline \multicolumn{4}{|l|}{ Sexo } \\
\hline Feminino $(n=121)$ & $79(70,5)$ & $42(52,5)$ & $0,0109^{*}$ \\
\hline Masculino $(n=71)$ & $33(29,5)$ & $38(47,5)$ & 0,0109* \\
\hline \multicolumn{4}{|l|}{ Local da queda } \\
\hline Domicílio $(n=152)$ & $97(86,6)$ & $55(68,8)$ & $0,0027^{*}$ \\
\hline Trabalho $(n=3)$ & $1(0,9)$ & $2(2,5)$ & 0,093 \\
\hline Via pública $(n=36)$ & $13(11,6)$ & $23(28,8)$ & $0,0026^{*}$ \\
\hline Asilo $(n=1)$ & $1(0,9)$ & 0 & 0,395 \\
\hline \multicolumn{4}{|l|}{ Desfecho } \\
\hline Alta $(n=187)$ & $110(98,2)$ & $77(96,3)$ & 0,414 \\
\hline Óbito $(n=5)$ & $2(1,8)$ & $3(3,8)$ & 0,394 \\
\hline \multicolumn{4}{|l|}{ Queda no banho } \\
\hline $\operatorname{Sim}(n=11)$ & $5(4,5)$ & $6(7,5)$ & 0,379 \\
\hline Não $(n=181)$ & $99(88,4)$ & $70(87,5)$ & 0,850 \\
\hline \multicolumn{4}{|c|}{ Hipertensão arterial sistêmica (HAS) } \\
\hline Não $(n=106)$ & $55(49,1)$ & $51(63,8)$ & $0,0434^{*}$ \\
\hline $\operatorname{Sim}(n=86)$ & $57(50,9)$ & $29(36,3)$ & $0,0449^{*}$ \\
\hline \multicolumn{4}{|l|}{ Diabetes mellitus (DM) } \\
\hline Não $(n=163)$ & $98(87,5)$ & $65(81,3)$ & 0,237 \\
\hline $\operatorname{Sim}(n=29)$ & $14(12,5)$ & $15(18,8)$ & 0,230 \\
\hline \multicolumn{4}{|c|}{ Acidente vascular cerebral (AVC) } \\
\hline Não $(n=184)$ & $109(97,3)$ & $75(93,8)$ & 0,231 \\
\hline $\operatorname{Sim}(n=8)$ & $3(2,7)$ & $5(6,3)$ & 0,220 \\
\hline \multicolumn{4}{|c|}{ Doença neurológica/psiquiátrica ${ }^{\mathrm{b}}$} \\
\hline Não $(n=161)$ & $101(90,2)$ & $60(75)$ & $0,0048^{*}$ \\
\hline $\operatorname{Sim}(n=31)$ & $11(9,8)$ & $20(25)$ & $0,0048^{*}$ \\
\hline \multicolumn{4}{|l|}{ Doença osteomuscularc } \\
\hline Não (n=189) & $111(99,1)$ & $78(97,5)$ & 0,379 \\
\hline $\operatorname{Sim}(n=3)$ & $1(0,9)$ & $2(2,5)$ & 0,067 \\
\hline
\end{tabular}

* p-valor significativo pelo teste Z considerando nível de significância de 5\%.

a'Dados ignorados não foram apresentados.

'Inclui: demência, ansiedade, depressão, mal de Alzheimer, mal de Parkinson, crise convulsiva, aneurisma e esclerose múltipla. Inclui: osteoporose, artrite, artrose.

Dentre os participantes, 115 (59,9\%) usavam algum tipo de medicamento e dentre estes 71 (63,4\%) idosos não tiveram TCE/TRM, ao passo que 44 (55\%) idosos apresentaram TCE/TRM. A relação entre o uso de medicamentos e a ocorrência de TCE/TRM em idosos que sofreram queda do mesmo nível consta na Tabela 3. 
Tabela 3 - Distribuição dos medicamentos associados à ocorrência de TCE e TRM em idosos que sofreram queda do mesmo nível atendidos no PA, 2014

\begin{tabular}{|c|c|c|c|}
\hline Medicamento & $\begin{array}{c}\text { Sem TCE/TRM } \\
\text { n (\%) }\end{array}$ & $\begin{array}{c}\text { Com TCE/TRM } \\
\text { n (\%) }\end{array}$ & p-valor \\
\hline \multicolumn{4}{|c|}{ Anti-hipertensivo } \\
\hline Não $(n=125)$ & $69(61,6)$ & $56(70)$ & 0,229 \\
\hline $\operatorname{Sim}(n=67)$ & $43(38,4)$ & $24(30)$ & 0,229 \\
\hline \multicolumn{4}{|l|}{ Psicotrópico } \\
\hline Não $(n=164)$ & $97(86,6)$ & $67(83,8)$ & 0,588 \\
\hline $\operatorname{Sim}(n=28)$ & $15(13,4)$ & $13(16,3)$ & 0,575 \\
\hline \multicolumn{4}{|c|}{ Insulina e Hipoglicemiante } \\
\hline Não $(n=175)$ & $103(92)$ & $72(90)$ & 0,630 \\
\hline $\operatorname{Sim}(n=17)$ & $9(8)$ & $8(10)$ & 0,630 \\
\hline \multicolumn{4}{|l|}{ Antiarrítmico } \\
\hline Não $(n=187)$ & $110(98,2)$ & $77(96,3)$ & 0,414 \\
\hline $\operatorname{Sim}(n=5)$ & $2(1,8)$ & $3(3,8)$ & 0,394 \\
\hline \multicolumn{4}{|l|}{ Analgésico } \\
\hline Não $(n=191)$ & $111(99,1)$ & $80(100)$ & 0,395 \\
\hline $\operatorname{Sim}(n=1)$ & $1(0,9)$ & $0(0)$ & 0,395 \\
\hline \multicolumn{4}{|c|}{ Terapia hormonal } \\
\hline Não $(n=188)$ & $109(97,3)$ & $79(98,8)$ & 0,755 \\
\hline $\operatorname{Sim}(n=4)$ & $3(2,7)$ & $1(1,3)$ & 0,506 \\
\hline
\end{tabular}

* p-valor não significativo pelo teste Z considerando nível de significância de 5\%.

\section{DISCUSSÃO}

Pelo presente estudo, verificou-se a incidência de TCE/TRM em idosos que tiverem algum tipo de complicação decorrente da queda do mesmo nível ( $n=80,41,7 \%)$. Contudo, torna-se difícil a quantificação da real dimensão do trauma no idoso, porque a incidência total das lesões é desconhecida, uma vez que muitas não são relatadas. Os índices de trauma são importantes na mensuração da gravidade das lesões e no cálculo da probabilidade de sobrevida, auxiliando na avaliação da qualidade do atendimento ${ }^{(14-15)}$.

No que tange à du ração e evolução, as lesões no paciente idoso são mais críticas e, consequentemente, consomem mais recursos e causam maior impacto na vida dos familiares. As quedas são um importante problema de saúde pública, em virtude de sua frequência, morbidade e elevado custo social e econômico decorrente das lesões provocadas ${ }^{(8)}$.

Cumpre mencionar que, no ano de 2017, no Brasil, as internações hospitalares urgentes financiadas pelo Sistema Único de Saúde (SUS) corresponderam a mais de 9 milhões, e destas $25 \%$ foram de indivíduos com 60 anos ou mais ${ }^{(16)}$.

Verificou-se no presente estudo que os idosos que apresentaram TCE/TRM chegaram ao hospital por intermédio de transporte especializado $(p=0,0001)$, enquanto os que não sofreram este agravo chegaram sozinhos, com familiares, ou com transporte terrestre do município de origem ( $p=0,0001)$.

O socorro e transporte especializado podem minimizar as possíveis consequências de um trauma, assim como denotar maior gravidade, como nos casos de TCE ou TRM. Porém, este deveria ser, senão o único, ao menos o tipo de transporte mais utilizado nas circunstâncias de queda e/ou outros tipos de trauma em idosos, tendo em vista que o socorro e transporte adequados podem minimizar as possíveis consequências do trauma.

Destaca-se que o acesso de idosos ao PA com história de trauma, sem o transporte especializado, 
tem sido verificado em outros estudos ${ }^{(17)}$. Desse modo, deve ser ressaltada a importância do serviço de atendimento pré-hospitalar móvel, cujos profissionais são capacitados para identificar e intervir em condições graves, bem como em condições específicas dos idosos. É importante um atendimento pré-hospitalar especializado com benefícios do rastreio e intervenção rápida nos casos de condições agudas e traumas, com transferência para serviços especializados que atendam às suas necessidades e contribuam para a redução da mortalidade e sequelas do trauma, evitando um primeiro atendimento tardio e inadequado(18).

As informações referentes à classificação de risco dos idosos foram baseadas em um protocolo que priorizava o atendimento conforme a indicação clínica, classificando-o em cores com tempo de espera diferenciado, para assim diminuir o número de mortes evitáveis, sequelas e internações ${ }^{(13)}$.

Embora se tenha verificado que pacientes sem TCE/TRM tenham se associado à classificação de urgência menor (verde), pacientes com TCE/TRM não apresentaram associação com classificação de risco em eixos mais graves (amarelo ou vermelho). Apesar disso, é importante ressaltar que os dados podem ter sido influenciados pelo número de pacientes ignorados, uma vez que o ACCR, na instituição, funcionava 18 horas por dia (7:00 horas até 01:00 hora).

A ausência de classificação de risco pode expor o paciente ao agravamento do quadro, uma vez que é feita com base em protocolos que levam em consideração critérios $\operatorname{clínicos}^{(13,19)}$. A classificação de risco visa identificar o paciente com potencial risco à vida, o que possibilita a ampliação da resolutividade dos eventos da doença e grau de sofrimento do usuário, quando o atendimento é prioritário. Isso significa que, neste estudo, embora alguns idosos que sofreram queda possam ter sido classificados como casos de atendimento de urgência menor, seus quadros clínicos podem ter se agravado posteriormente.

Quanto ao tempo de internação, após o trauma, o idoso permanece internado por mais tempo que o adulto jovem ${ }^{(20)}$. Em contrapartida, estudo realizado com idosos e não idosos, vítimas de trauma, no município de Curitiba-PR, mostrou não haver diferença significativa entre os grupos, com uma média de 7,66 dias de internação ${ }^{(21)}$. No presente estudo foi constatado que o tempo de internação em idosos que sofreram queda do mesmo nível não estava associado ao TCE/TRM, e o tempo de internação foi de 3,9 $\pm 3,8$ dias entre estes indivíduos.

Considerando a mortalidade, embora não tenha sido verificada diferença estatística, a proporção de óbitos foi maior entre os pacientes que tiveram TCE/TRM. O número de óbitos pode não representar a real situação, uma vez que houve a transferência de pacientes para outras instituições hospitalares, das quais não se sabe o desfecho clínico.

Estudos mostram que, no trauma, as lesões são mais graves em idosos se comparadas ao adulto jovem, assim como a necessidade de Unidade de Terapia Intensiva (UTI), o tempo de internação e a taxa de mortalidade ${ }^{(20,22)}$. Assim, o trauma e suas sequelas tornaram-se um dos principais problemas de saúde pública, com incidência e mortalidade crescentes.

Quanto ao sexo, verifica-se, na Tabela 2, que em pacientes do sexo feminino há uma maior prevalência de queda sem TCE/TRM, ao passo que pessoas do sexo masculino apresentaram maior prevalência de queda com TCE/TRM.

Estudo realizado em São José do Rio Preto-SP(23), com 535 idosos vítimas de trauma, mostra que o número de quedas foi de 373(69,7\%), prevalecendo também o sexo feminino 192 (52,4\%).

A maior frequência de quedas sofridas por pessoas do sexo feminino pode ser explicada por estas apresentarem maior fragilidade, serem mais ativas dentro e fora do lar e pela prevalência de doenças crônicas ${ }^{(24)}$.

Observaram-se resultados com significância estatística entre os idosos que tiveram e não tiveram TCE/TRM quanto ao sexo, presença de HAS e doença psiquiátrica; isso pode significar que estas variáveis são associadas à ocorrência de queda em idosos, mas não têm influência sobre a ocorrência de TCE/TRM. Verificou-se, por exemplo, que os pacientes sem HAS apresentaram maior prevalência de queda com TCE/TRM, enquanto que nos pacientes com HAS havia prevalência de queda sem TCE/TRM.

Quanto às doenças neurológicas e psiquiátricas, observou-se que os pacientes com doença 
neurológica apresentaram maior prevalência de quedas com TCE/TRM e aqueles sem a doença neurológica tiveram maior prevalência de queda sem TCE/TRM. Isto pode ser explicado pelas limitações físicas e mentais características desse tipo de doença, que torna a marcha e o equilíbrio do idoso instável, por conseguinte, deixando-os mais propensos a episódios de queda.

Vários estudos destacam a ocorrência da maioria das quedas no domicílio ${ }^{(5,16)}$, porém não revelam se estas resultam em TCE ou TRM. Um dos fatores que podem contribuir para que a queda ocorra no lar é a falta de adaptação do ambiente para o idoso, com pisos escorregadios, tapetes, escadas, ou seja, as alterações e limitações inerentes ao envelhecimento não são levadas em consideração.

A queda do mesmo nível pode estar relacionada não só às alterações físicas e limitações do envelhecimento. Associar esses fatores ao domicílio aponta para a necessidade de maior atuação da equipe de saúde da atenção primária, representada pelos integrantes da Estratégia Saúde da Família, com vistas à identificação de fatores ambientais que favoreçam a ocorrência de quedas e, ainda, orientação aos idosos e residentes quanto ao manejo adequado destes fatores, como é o caso dos tapetes. Ademais, também é possível intervir ou promover educação preventiva sobre práticas individuais cotidianas que devem ser evitadas, pois contribuem para a queda, por exemplo, andar dentro de casa no escuro.

Quanto aos medicamentos de uso contínuo dos idosos que sofreram queda, não foi observada associação do uso medicamentoso com a queda, haja vista que o uso de medicamento segue o mesmo padrão entre todos os idosos, ou seja, a maioria dos pacientes com TCE/TRM não fazem uso das várias classes de medicação mencionadas. Vários autores afirmam que as quedas sofridas por idosos estão relacionadas diretamente com as doenças crônicas e a polifarmácia, as quais interferem na condição respiratória e hemodinâmica do idoso, mascarando, por vezes, a gravidade do trauma e iniciando quadros de hipoperfusão e hipóxia ${ }^{(25)}$. Não só a polifarmácia, mas a adesão incorreta, interações medicamentosas e efeitos adversos devem ser analisados no idoso.

Embora nesta pesquisa não tenha sido identificada influência do uso de medicamentos na ocorrência ou não de TCE/TRM, esta prática é considerada um fator de risco importante para quedas de idosos, principalmente quando se trata de medicamentos indicados para doenças cardiovasculares, insulina, antiinflamatórios não esteróides e antiepilépticos, em virtude de seus efeitos colaterais cumulativos, da interação com outras drogas ou da hipotensão ortostática ${ }^{(3)}$. Por isso, a orientação quanto ao uso desses medicamentos deve ser estritamente indicada pelo médico e acompanhada pelos profissionais de saúde.

O estudo teve como limitações uma população pequena e a ausência do registro de informações nos prontuários.

\section{- CONCLUSÃO}

As características do trauma sofrido por idosos são bem estabelecidas e a causa de trauma mais frequente é a queda do mesmo nível, principalmente entre as mulheres. Nesse cenário, a incidência de lesões como TCE e TRM é elevada e pode impactar negativamente a qualidade de vida do idoso, de sua família, e onerar o sistema de saúde.

Neste estudo verificou-se que os idosos com TCE/TRM chegaram ao hospital por transporte especializado. Pacientes classificados de urgência menor (verde) não tinham TCE/TRM, mas a ausência de associação do TCE/TRM com emergência (vermelho) pode ser consequência do horário limitado de funcionamento do ACCR ou ainda, do agravamento do quadro após a classificação de risco.

Embora não tenha sido verificada diferença estatística, a proporção de óbitos foi maior entre os pacientes que tiveram TCE/TRM.

Embora as alterações fisiológicas do envelhecimento tornem os idosos mais suscetíveis à queda e a prognósticos ruins, neste estudo não foi verificado aumento do tempo de internação.

Essas informações são de grande importância para a saúde pública, no que concerne à elaboração de estratégias que visam a medidas educativas e preventivas, seja de forma individual ou coletiva, 
que integrem políticas, a fim de reduzir a incidência de traumas sofridos por idosos e diminuir a morbimortalidade.

\section{- REFERÊNCIAS}

1. Kramarow E. Chen L, Hedegaard H, Warner M. Deaths From Unintentional Injury Among Adults Aged 65 and Over: United States, 2000-2013. Centers for Disease Control and Prevention. [Internet]. 2015 [acesso em 2018 maio 08]; 199 .Disponível em: https://www.cdc.gov/nchs/data/databriefs/db199.pdf.

2. Sherrington C, Tiedemann A. Physiotherapy in the prevention of falls in older people. Physiotherapy in the prevention of falls in older people. Journal of Physiotherapy. [Internet]. 2015 [acesso em 2015 out 14 ]; 61. Disponível em: http://www.journalofphysiotherapy.com/article/S1836-9553(15)00012-0/pdf.

3. Ambrose AF, Paul G, Hausdorff JM. Risk factors for falls among older adults: a review of the literature. Maturitas. [Internet]. 2013 [acesso em 2016 ago 05]; 75(1). Disponível em: https://doi.org/10.1016/j.maturitas.2013.02.009.

4. Jagnoor J, Keay L, Jaswal N, Kaur M, Ivers R. A qualitative study on the perceptions of preventing falls as a health priority among older people in Northern India. Inj Prev. [Internet]. 2014 [acesso em 2014 out 05]; 20(1). Disponível em: https://doi.org/10.1136/injuryprev-2012-040707.

5. Freitas MG, Bonolo PF, Moraes ENM, Machado CJ. Idosos atendidos em serviços de urgência no Brasil: um estudo para vítimas de quedas e de acidentes de trânsito. Ciênc. saúde coletiva. [Internet]. 2015 [acesso em 2018 maio 08]; 20(3). Disponível em:https://doi.org/10.1590/1413-81232015203.19582014.

6. Li YH, Song, GX, Yu Y, Zhou D, Zang HW. Study on age and education level and their relationship with fallrelated injuries in Shanghai, China. BiomedEnvironSci.[Internet]. 2013 [acesso em 2014 jun 05]; 26(2). Disponível em: http://www.besjournal.com/Articles/Archive/2013/No2/201301/t20130116_76366.html.

7. Karlsson MK, Magnusson H, Von ST, Rosengren BE. Prevention of falls in the elderly-a review. Osteoporos. [Internet]. 2013 [acesso em 2015 jun 27] ;24(3). Disponível em: https://doi.org/10.1007/s00198-012-2256-7.

8. MarliesRJ, Elst MV, Hartholt KA. Drug-related falls in older patients: implicated drugs, consequences, and possible prevention strategies. Ther. Adv. DrugSaf. [Internet]. 2013 [acesso em 2014 set 14]; 4(4). Disponível em: https://doi.org/10.1177/2042098613486829.

9. Abbasi, HR, Mousavi SM, Akerdi T, Niakan MH, Bolandparvaz S, Paydar S. Pattern of traumatic injuries and Injury Severity Score in a major trauma center in Shiraz, Southern Iran. Bull Emerg Trauma. [Internet]. 2013 [acesso em 2014 jul 20]; 1(2). Disponível em: https://www.ncbi.nlm.nih.gov/pubmed/27162829.

10. Silva LAP, Ferreira AC, Paulino RES, Guedes GO, da Cunha MEB, Peixoto VTCP, Faria TA. Análise retrospectiva da prevalência e do perfil epidemiológico dos pacientes vítimas de trauma em um hospital secundário. Rev Med. [Internet]. 2017 [acesso em 2018 mar 18]; 96(4). Disponível em: https://www.revistas.usp.br/revistadc/article/ view/127308/136722.

11. Costa ACC, Fortesa RC. Idosos vítimas de quedas admitidos na terapia intensiva: um estudo retrospectivo analítico. GeriatrGerontolAging,[Internet]. 2016 [acesso em 2018 maio 08]; 10(4). Disponível em: http://www. ggaging.com/details/399/en-US/idosos-vitimas-de-quedas-admitidos-na-terapia-intensiva--um-estudoretrospectivo-analitico. 
14. Silva RA, Araujo MZ, Tacio R, da Silva J, Pereira BBM, Medeiros AC. O traumatismo craniano encefálico moderado e grave. INTESA. [Internet]. 2015 [acesso em 2016 ago 08]; 9(1). Disponível em: http://www.gvaa.com. br/revista/index.php/INTESA/article/view/3245/3203.

15. Degani GC, Pereira Júnior GA, Rodrigues RAP, Luchesi BM, Marques S. Idosos vítimas de trauma: doenças preexistentes, medicamentos em uso no domicílio e índices de trauma. Rev. Bras. Enf. [Internet]. 2014 [acesso em 2018 abr 20]; 67(5). Disponível em: http://dx.doi.org/10.1590/0034-7167.2014670513.

16. Ministério da Saúde (BR). Datasus. Morbidade hospitalar por local de internação. [Internet] Brasília: Ministério da Saúde; 2017. [acesso em 2017 abr 19]. Disponível em: http://tabnet.datasus.gov.br/cgi/tabcgi.exe?sih/cnv/niuf. def.

17. Rodrigues J, Mantovani MF, Ciosak SI. O idoso e o trauma: perfil e os fatores desencadeantes. Revenferm UFPE. [Internet]. 2015 [acesso em 2018 fev 07]; 9(3). Disponível em: https://periodicos.ufpe.br/revistas/ revistaenfermagem/article/view/10436/11238.

18. Carpenter CR, Platts-Mills TF. Evolving prehospital, emergency department, and "inpatient" management models for geriatric emergencies. Clinics in GeriatricMedicine. [Internet]. 2013 [acesso em 2017 ago 20]; 29(1). Disponível em: http://dx.doi.org/10.1016/j.cger.2012.09.003.

19. Duro CLM, Lima MADS, Levandovski PF, Bohn MLS, Abreu KP. Percepção de enfermeiros sobre a classificação de risco em unidades de pronto atendimento. Rev Rene 2014 [acesso em 2018 abr 20]. Disponível em: https:// www.lume.ufrgs.br/bitstream/handle/10183/106865/000938073.pdf? sequence=1.

20. Rosen T, Clark S, Bloemen EM, Mulcare MR, Stern ME, Hall JE. et al. Geriatric Assault Victims Treated at U.S. Trauma Centers: FiveYear Analysis of the National Trauma Data Bank. Injury. [Internet]. 2016 [acesso em 2018 maio 9]; 47(12). Disponível em: http://dx.doi.org/10.1016/j.injury.2016.09.001.

21. Broska Júnior CA, Folchini AB, Ruediger RR. Estudo comparativo entre o trauma em idosos e não idosos atendidos em um hospital universitário de Curitiba. Rev. Col. Bras. Cir. [Internet]. 2013 [acesso em 2016 jul 20]; 40(4). Disponível em: http://dx.doi.org/10.1590/S0100-69912013000400005.

22.Demontiero O, Gunawardene P, Duque G. Postoperative Prevention of Fallsin Older Adults with Fragility Fractures.ClinGeriatr Med. [Internet]. 2014 [acesso em 2018 maio 09]; 30(2). Disponível em: http://dx.doi. org/10.1016/j.cger.2014.01.018.

23. Oliveira KA, Rodrigues CC, Ribeiro RCHM, Martins CS, Abelan US, Fernandes AB. Causas de traumas em pacientes idosos atendidos em unidade de emergência. J Nurs UFPE. [Internet]. 2013 [acesso em 2016 jul 20]; 7(4). Disponível em: https://periodicos.ufpe.br/revistas/revistaenfermagem/article/view/11587.

24. Santos RKM, Maciel ACM, Britto HMJS, Lima CCL, Souza TO. Prevalência e fatores associados ao risco de quedas em idosos adscritos a uma Unidade Básica de Saúde do município de Natal, RN, Brasil. Ciênc. saúde coletiva.[Internet]. 2015 [acesso em 2018 maio 09]; 20(12). Disponível em: https://doi.org/10.1590/1413812320152012.00662015 .

25. Fhon JRS, Rosset I, Freitas CP, Silva AO, Santos JLF, Rodrigues RAP. Prevalência de quedas de idosos em situação de fragilidade. Rev Saúde Públ. [Internet]. 2013 [acesso em 2016 ago 12]; 47(2). Disponível em: http:// www.periodicos.usp.br/rsp/article/view/76629. 